
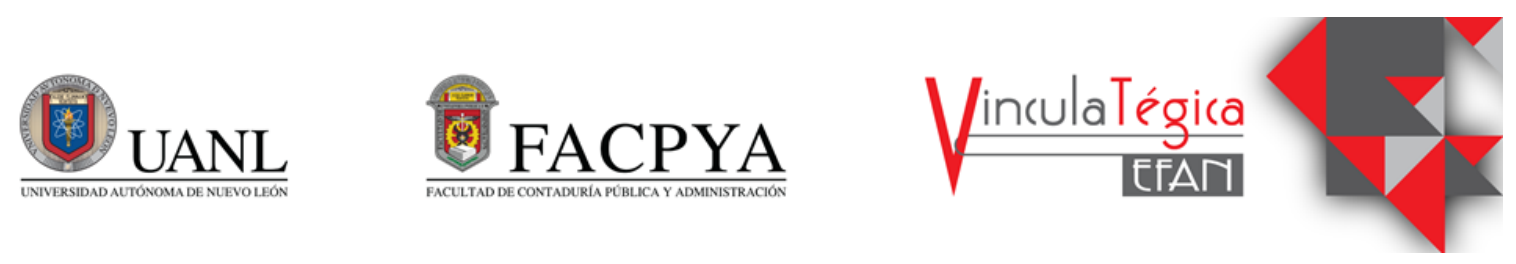

\title{
Validación de un instrumento de investigación para determinar un plan de marketing digital estratégico con adopción en TIC de vanguardia para posicionar a las PyMES de comercio
}

\author{
María de la Luz Medina Gracia ${ }^{1}$, Francisco Canibe Cruz $^{2}$ y Martín Jaramillo Rosales ${ }^{3}$ \\ 1, 2 y ${ }^{3}$ Universidad Autónoma de Coahuila, Facultad de Contaduría y Administración, Unidad Torreón, \\ majdwlin@hotmail.com,fcanibe@uadec.edu.mx,martinjaramillorosa@uadec.edu.mx, \\ Boulevard Revolución No. 151 Ote. Torreón, Coahuila, México, Tel. 01(871) 712 8044, Ext. 117
}

Información del artículo revisado por pares

Fecha de aceptación: junio-2021

Fecha de publicación en línea: diciembre-2021

DOI: https://doi.org/10.29105/vtga7.1-96

\begin{abstract}
Resumen
El aplicar una evaluación de plan de marketing digital, es de suma importancia para conocer la capacidad de que la empresa tome nuevas formas de comercialización con innovación estratégica, ya que es casi imprescindible que un negocio se encuentre en Internet. El Plan de marketing estratégico con adopción en TIC de vanguardia, apoya una gestión adecuada para poder posicionarse en el mercado, así como mostrar la necesidad de contar con esto. El propósito de este trabajo es validar un instrumento para determinar el grado de adopción, el impacto y la adaptación de la implementación de un plan de marketing digital estratégico con adopción en TIC de vanguardia para posicionar a las Pyme del Sector Comercio. Se acoplaron ocho instrumentos sobre el plan de marketing con adopción en TIC de vanguardia, resultando 4 dimensiones.
\end{abstract}

Palabras clave: plan, marketing, estrategia, adopción, instrumento de investigación, TIC.

\section{INTRODUCCIÓN}

Un acontecimiento importante es como el marketing digital se ha transformado en los últimos años más dinámicamente (Núñez y Corrales, 2020), ya que gran parte del mundo está pasando una considerable cantidad de tiempo confinado, los usuarios de las redes sociales han crecido con la mayor rapidez

\begin{abstract}
Applying a digital marketing plan evaluation is of utmost importance to know the ability of the company to take new forms of marketing with strategic innovation, since it is almost essential that a business is online. The Strategic Marketing Plan with adoption of cutting-edge ICTs supports proper management to position itself in the market, as well as showing the need for this. The purpose of this work is to validate an instrument to determine the degree of adoption, impact, and adaptation of the implementation of a strategic digital marketing plan with adoption of cutting-edge ICT to position SMEs in the Commerce Sector. Eight instruments were coupled on the marketing plan with adoption of cutting-edge ICT, resulting in 4 dimensions.
\end{abstract}

Keywords: plan, marketing, strategy, adoption, research instrument, TIC.

(Álvarez, 2021), por otro lado García (2021) menciona que con la llegada del Covid-19 y el encierro, las redes sociales se han convertido, en el medio de comunicación de las personas hacía el exterior.

La pandemia impulsó el uso de redes sociales, este 2021, su uso llegó a la mitad de la población, con un total del $53.6 \%$, es decir 4.200 millones (Galeano, 2021). Por lo 
anterior el uso de las tecnologías de la información y la comunicación ha aumentado por las empresas con el fin de presentar sus productos y conocer a sus clientes (Angamarca, Mena, Silva y Ballesteros, 2019).

Por lo anterior los líderes empresariales deben incorporar estrategias de marketing digital en sus planes comerciales para reducir la probabilidad de fracasar y hacer crecer su negocio (Junusi, 2020). Para Mejía (2019) es necesario desarrollar liderazgo estratégico para el marketing digital. De igual forma el marketing digital permite que los clientes potenciales encuentren en una diversidad de productos y servicios en redes sociales, donde el usuario se sienta atraído por que se ofrece (Terán, Espinosa, Márquez, Hernández, Ruíz y Vergara, 2018).

El Plan de Marketing digital es una herramienta de planificación, que implica desde la orientación al mercado, al segmento de clientes tanto actuales como potenciales, estudiar a la competencia, y las estrategias a utilizar (Armijos, 2018). A pesar de que el marketing en redes sociales pueda ser gratuito, es que la única manera de obtener beneficios es elaborar un plan de marketing que esquematice todo lo que la compañía quiere lograr (Sánchez y Tena, 2019).

La presente investigación formula que los negocios en México tienen impedimentos como la falta de recursos tecnológicos, es decir una cultura de la adopción de las tecnologías (Kato, 2019) para poder implementar páginas Web con un plan de marketing digital que permita posicionar su comercialización a mayor escala (Sánchez y Tena, 2019) y debido al atraso en el uso de estas, el miedo y falta de emprendimiento, no cuentan con página web, ni redes sociales, que puedan atraer a sus clientes (Terán et al., 2018).

El estudio tiene como objetivo determinar el grado de adopción, el impacto y la adaptación de la implementación de un plan de marketing digital estratégico con adopción en TIC de vanguardia para posicionar a las Pyme del Sector Comercio, por consecuencia se desprenden las siguientes interrogantes ¿Cómo influye un plan de marketing digital con enfoque de la adopción de herramientas tecnológicas de vanguardia?, ¿De qué manera contribuye la cultura de la adopción de las herramientas tecnológicas en un plan de marketing digital?, ¿Existe efecto positivo en la rentabilidad en las empresas del sector de servicio en función del marketing digital?.

Madrigal, Alfaro y Madrigal (2018) afirman que en México el $45 \%$ de usuarios siguen alguna marca en Internet, respecto al uso de redes sociales, el 95\% de los usuarios cuenta con Facebook, el 93\% tiene WhatsApp y el $72 \%$ utiliza el YouTube.

Debido a lo anterior la investigación se enfoca en MiPymes de la ciudad de Torreón, en el estado de Coahuila, México, durante el año 2021. De acuerdo a González y Moreno (2020) los negocios abiertos dan un total de 1018 , teniendo como sector comercio $44.01 \%$, servicios $50.39 \%$, manufactura $5.11 \%$, construcción $0.49 \%$, microempresas $94.93 \%$

El Plan de marketing digital va dirigido a los dueños o administradores de MiPymes para una gestión eficiente, al dar a conocer su producto o servicio, al contar con el conocimiento de las herramientas de vanguardia para su negocio.

Por consiguiente, la contribución es proporcionar un instrumento de medición para un plan de marketing digital estratégico y una prueba empírica para el modelo (Mitręga, 2019).

El artículo se encuentra estructurado de la siguiente forma: en primer lugar, se realiza una revisión de la literatura sobre planes de marketing digitales estratégicos; en segundo lugar, se plasma la metodología de la 
investigación y se determinan las características de la muestra; en tercer lugar, se aplica el método de expertos en donde se determina el nivel de competencia, se aplica un pilotaje para validar el instrumento de investigación y por último se exponen las principales conclusiones y recomendaciones.

\section{MARCO TEÓRICO}

2.1 Influencia de un plan de marketing digital con enfoque de la adopción de herramientas tecnológicas de vanguardia.

Amoah \& Bashiru (2021) relaciona que, con la globalización, se provocaron cambios en la tecnología, creando posibilidades y desafíos estratégicos para las PYME mediante la integración a las redes sociales como una nueva herramienta de marketing y un cambio de paradigma. Con la influencia de aspectos externos como cambios principales de políticas de gobierno y economía mundial, es imprescindible el aplicar una adecuada planificación estratégica de marketing para un buen desenvolvimiento empresarial (Jaramillo, Tacuri y Trelles, 2018). Los mercados actuales en los que los clientes están más informados y son cada vez más exigentes, la existencia de una libre competencia y el rápido desarrollo tecnológico, es necesario que el marketing cuente con una dimensión estratégica (Juárez, 2017). La rapidez con que se obtiene información, pues la tecnología permite en tiempo real tener y usar la información (More, Castillo y Rodríguez, 2018).

Debido al éxito de programas de análisis de marketing, las organizaciones tienen un efecto profundo en la tasa de adopción (Cates, 2018). Morán y Cañarte (2017) afirman que las redes sociales son indispensables en las estrategias de marketing, porque trata de la atracción y fidelización de clientes y además, crea una experiencia más personalizada basada en diferentes parámetros, como, ingresos, edad, sexo, ubicación y compras (Grandhi, Patwa \& Saleem, 2020), así como medir la popularidad en función del nivel de actividad que presentan sus miembros (Narváez \& Montalvo, 2014).

El uso de las múltiples plataformas existentes ha logrado facilitar la participación e interacción de los usuarios con las empresas que implementan el marketing digital (Lozano y Torres, 2017). Por lo anterior las empresas con la tecnología, realizan un seguimiento de los diferentes análisis relacionados con las plataformas como Facebook, Twitter, WhatsApp, Instagram, YouTube (Alameddine, 2013; Narváez \& Montalvo 2014; Dirgiatmo, 2015; Calle, Erazo y Narváez, 2020). De modo que Taiminen \& Karjaluoto (2015) consideran que a algunos productos y servicios simplemente se comunican mejor a través de medios digitales, además de las presiones externas como la competencia y la necesidad expandir mercados, en consecuencia las pymes intentan adoptar tecnologías con el fin de aprovechar las ventajas que éstas les aportan y adaptarse a los cambios constantes (Arellano, Romero y Guzmán, 2018).

El marketing digital como señala Maridueña y Paredes (2015), es un conjunto de instrumentos desde páginas web, correos electrónicos y otras herramientas basadas en el uso del Internet, que a su vez se puede medir el impacto de las acciones sobre un producto y servicios, ahora se ha convertido en un instrumento de apoyo estratégico a la organización (Núñez y Corrales, 2020) y desde el punto de vista de Armijos (2018); Correa (2019), es relevante la marca, que se difunde en la web; el logo, para tener presencia; el slogan, que es el mensaje correcto, a la persona correcta y el tiempo correcto con el manejo de las herramientas del marketing digital, para esto, González y Moreno (2020) manifiestan que se debe elaborar una serie de acciones planificadas que 
faciliten la toma de decisiones para lograr un objetivo con mayores rendimientos $\mathrm{y}$ beneficios, lo mismo ocurre para identificar y optimizar los posibles problemas que puedan ocurrir en las diferentes fases de atracción de un cliente, con el fin de medir los resultados (Vidal, 2016), así como comprender las necesidades de sus clientes, el tipo de alternativas disponibles, y los atributos clave en el impacto de las experiencias digitales (Kaur, 2020). Por estas razones Brown, Foroudi \& Hafeez (2019) relaciona que los gerentes pueden implementar una guía para las acciones, con una gestión del desempeño de la empresa que está alineada con las necesidades comerciales actuales. Hänninen, Kwan \& Mitronen (2021) considera que los comercios comenzaron a compartir ofertas y descuentos en redes sociales, empezaron a crecer, sin embargo, en muchas tiendas físicas disminuyó la afluencia, ya que continuaban dependiendo de los medios tradicionales para dirigir el tráfico a la tienda.

\subsection{Contribución de la cultura de la adopción de las herramientas tecnológicas en un plan de marketing digital}

El impacto de la tecnología es mayor, la oportunidad de ofrecer información en tiempo real a los usuarios, a los cuales pretenden llegar cada una de las Pymes del sector comercio por medio de los distintas herramientas (Lozano y Torres, 2017), de ahí que uno de los nuevos avances que inevitablemente tendrán que realizar los comercios, es la expansión del mercado a través del online, donde se demostró su eficacia, para poder penetrar en el mercado Nacional e internacional (Pangemanan \& Walukow, 2018). Las Pymes tanto para tener presencia físicas, como virtualmente, deben utilizar la difusión por medios digitales con las herramientas existentes y deben tener un espacio dentro de la estructura organizativa que piensa en el posicionamiento de su marca (Hoyos y Sastoque, 2020). A su vez se ha vuelto casi un requisito para las Pymes que quieren crecer en el mercado, muchas de las empresas optaron por adoptar estas herramientas y de esta manera ser más competitivos (Torres, Rivera, Cabarcas y Castro, 2017). Un estudio realizado de redes sociales en línea, en Corea, demostró que existe un impacto significativo de compras de amigos. Este comportamiento social podría llamar significativamente la atención de los gerentes, ya que puede brindar una nueva oportunidad de marketing (Narváez \& Montalvo, 2014), de tal manera que el comportamiento del cliente también es un desarrollo que las empresas deben seguir (Taiminen \& Karjaluoto, 2015). La mentalidad del empresario para el marketing es localizar nuevas oportunidades, con la ayuda de la tecnología y reducción de costos (Juárez, 2017), así que Arellano et al., (2018) destaca que la reputación o imagen que se genera con las herramientas que ofrecen en Internet, se convierten en un instrumento para los cambios que existen en los sistemas de comunicación tradicionales, que permiten generar retroalimentación real y directa.

En el caso de La planeación estratégica de marketing es fundamental, ya que se definen, anticipan, crean y satisfacen las necesidades de los clientes (Juárez, 2017), no es solo un desempeño específico relacionado con la venta de productos y servicios, sino también un proceso de gestión de la relación entre la organización y el cliente (Khajehasani, Abolizadeh, \& Dehyadegari,, 2020), por eso, implica que cada decisión sea coherente entre sí con el análisis y el diagnóstico realizado, desde la orientación al mercado, a clientes actuales, potenciales, competencia, segmentación y estrategias a utilizar (Armijos, 2018) y es imprescindible que la implementación ayude a las empresas al 
cumplimiento y eficacia de los objetivos planteados (Jaramillo et al., 2018). Para la determinación de los objetivos en función de modelo SMART (específicos, medibles, alcanzables, relevantes y acotados en el tiempo), se pretende seleccionar no solo las áreas de trabajo implicadas sino también las herramientas del marketing (Segarra, Martínez, y Gauchi 2018).

El marketing digital no es solo un desempeño específico relacionado con la venta de productos y servicios, sino también un proceso de gestión de la relación entre la organización y el cliente (Khajehasani et al., 2020), exige nuevas formas de gestionar la administración, incidiendo de manera directa en el apoyo estratégico a (Núñez y Corrales, 2020), afronta un momento crucial, en el que nuevos enfoques intentan redefinir sus actividades, orientándolo hacia metodologías que, más allá de los aspectos comerciales, se preocupen por establecer nuevos mecanismos de comunicación con las personas (Sánchez, García y Rodríguez, 2020). Las empresas deben tomar en cuenta la orientación de la campaña, ya que el marketing digital siempre tiene algunos anuncios que no acaban siendo vistos por el usuario, y se consideran un despilfarro de presupuesto (Gupta \& Chokshi, 2020).

Así como los usuarios pasar más tiempo en redes sociales que en cualquier otro sitio, y han influido en como cambiar las actividades diarias de los consumidores, las empresas deben aprovechar la tecnología para expandir el alcance de sus clientes (Alameddine, 2013), también pueden determinar la interacción y comportamiento de los clientes, y el posicionamiento en buscadores, lo anterior evidencio el éxito de la utilización de las herramientas digitales (Calle et al., 2020). Para la empresa se trata de conseguir la captación de clientes que están presentes en las distintas redes sociales pero que aún no nos conocen (Morán y Cañarte, 2017).

Crear una plataforma en donde los usuarios puedan dejar sus ideas y comentarios, lo hace viable para mejorar la organización (González y Moreno, 2020). Dada la importancia del proceso, control y seguimiento de las acciones, que permitirá obtener una retroalimentación que ayude a implementar nuevos sistemas de mejora y a corregir o fortalecer aquellos elementos del plan en los deba haber cambios (Vidal, 2016). Los sitios web de marketing más elaborados no hacen desaparecer a los sitios web pequeños, y eso les permiten obtener una ventaja competitiva mediante la estrategia de segmentación del mercado (Khajehasani et al., 2020). Grandhi et al. (2020) define como líderes contendientes a quienes tienen una baja inversión continua, con aspiraciones a la alta inversión, por lo que es más común la medición de datos y toma de decisiones de marketing. A su vez mediante las redes sociales por grupos específicos se puede recopilar información de nuevos clientes y al mismo tiempo usar este tipo de canal para realizar ofertas y promociones exclusivas para potenciar un segmento específico (Maridueña y Paredes, 2015).

\subsection{Efecto en la rentabilidad en las empresas del sector comercio en función del marketing digital}

Las empresas que han adoptado el marketing digital $\mathrm{y}$ han realizado las inversiones necesarias, han podido aprovechar estas herramientas (Taiminen \& Karjaluoto, 2015). Como lo hace notar Torres et al., (2017) en las empresas hay buenos resultados en cuanto al cambio de comunicación de valor, a los clientes para implantar relaciones duraderas entre los usuarios y las Pymes permitiendo así un aumento de la rentabilidad y gratitud de las mismas. Cates (2018) declara que, en el 
comercio minorista, encontraron mejoras para las operaciones, los ingresos y las ganancias de organizaciones que han implementado marketing. Aumentan las posibilidades de ventas y clics incrementalmente por ofertas basadas en productos individualizados, y las recomendaciones (Kaur, 2020).

De acuerdo a Londoño, Mora y Valencia, (2018) el marketing digital es una nueva cultura que está generando beneficios económicos para el comercio. Por lo tanto las empresas deben tener los objetivos claros para definir canales y tácticas de comunicación para ingresar en los mercados, mejorando la rentabilidad y optimizar las inversiones realizadas en los negocios (Armijos, 2018). Con la finalidad de mejorar la rentabilidad, las grandes empresas implementan estrategias de marketing digital en sus actividades comerciales (Calle et al., 2020), así mismo para Lizama, Matos y Beltrán (2020) el online marketing es una alternativa económica para lograr mediante estrategias, incrementar el lucro en la empresa, en tanto permite el máximo aprovechamiento de los recursos tecnológicos con los que cuentan las MiPymes. Las empresas aplican estrategias digitales con la finalidad de mejorar la rentabilidad, competitividad y posicionamiento mediante el uso de diferentes redes sociales (Calle et al., 2020).

El diseño de la estrategia de redes sociales si favorece al incremento de ventas de la empresa (González y Moreno, 2020), dado que las referencias son decisivas para la generación de nuevas ventas de una empresa, la estrategia de marketing se puede construir en torno al cuidado de los clientes existentes (Alameddine, 2013). La información efectiva de redes sociales, para obtener previsiones de ventas, les permite diseñar campañas relevantes para retener y obtener clientes con el fin de mejorar su satisfacción y con esto tener mayor rentabilidad (Maloletko \&
Kaurova, 2021). También se consideró que el uso de las redes sociales cumplía el objetivo principal de aumentar las ventas (Taiminen \& Karjaluoto, 2015). Las redes sociales al ser un intermediario para el boca a boca donde los miembros pueden influir en el comportamiento de los demás, serán la acción - consecuencia que atraiga más ventas e ingresos para el vendedor (Narváez \& Montalvo, 2014).

Es relevante cómo la tecnología impacta de manera significativa en la comercialización y la utilización de redes sociales (Correa, 2019). El rendimiento del marketing electrónico desempeña un papel de intermediario entre las diferentes partes de la empresa, incluidos los sectores financieros (Khajehasani et al., 2020). El Éxito podría ser el retorno de la inversión, rentabilidad, efectividad, confiabilidad, utilidad o ventaja competitiva (Phippen, Sheppard, \& Furnell, 2004).

Las empresas gestionan eficazmente su programas de comunicación de marketing, cuando los empleados están envueltos de una manera que proyectan la imagen o la marca (Brown et al., 2019). Por lo anterior aumenta la eficiencia y la rentabilidad inversión (Grandhi et al., 2020). Se tiene que tener en cuenta el impacto de los canales digitales sobre la rentabilidad centrando la atención en las motivaciones y necesidades del cliente (Hänninen et al., 2021)

Jaramillo et al., (2018) reconocen la necesidad de la planeación estratégica de marketing para su crecimiento y bienestar a largo plazo, ayudando a orientar los recursos hacia las características de alta prioridad, comparado con Amoah \& Bashiru (2021) que relaciona que se pudieran eliminar los costos de beneficios por los ingresos, por la generación, atracción de clientes, aumento conciencia de marca, lealtad y reputación de la imagen en la Pymes, si no se cuenta con una 
planificación adecuada.

\subsection{Necesidad de evaluar el marketing digital}

Para Stanton (1987) "Un programa de evaluación total que consiste en una revisión sistemática, objetiva y total de la función de mercadotecnia. Una evaluación de metas, políticas y resultados, organización, personal y prácticas de la compañía" (p.742). Al no implementar auditorías de marketing como herramienta estratégica, las empresas presentan limitaciones para detectar las situaciones que prevalecen, como la competencia que cada vez es más agresiva, los cambios en los hábitos del consumidor, la falta de coordinación en los programas de marketing, originando soluciones inadecuadas o metas irreales (Oquendo, 2016).

Evaluar las estrategias permitirá entender la secuencia a aplicar de las empresas que están en crecimiento y reformular cambios basándose en los resultados (Noreña \& Álvarez, 2020).

\section{MÉTODO}

La metodología consta de la revisión de la literatura, el diseño del instrumento, la valuación por el método de expertos, la prueba piloto, pruebas de fiabilidad $\mathrm{y}$ validez, obtención de la muestra y aplicación del instrumento, el procesamiento de los datos, el resultado y las conclusiones. En la ilustración 1 se muestra el diseño de la metodología.

El presente estudio tiene un enfoque cuantitativo (Hernández, Fernández, Baptista, 2014), que utilizan medidas que captan solo porciones del concepto estudiado (Vara, 2012), ya que los datos recolectados están basados en mediciones numéricas. El tipo de metodología que se utilizan es exploratoria, descriptiva, transversal y correlacional para identificar los factores para la evaluación de un plan de marketing digital con herramientas de vanguardia, como lo sustentan Hernández, Méndez y Contreras, et al. (2014). Se genera una revisión de la literatura sobre el tema de investigación en bases de datos Scopus, Web of Science (WoS), artículos de revistas científicas indexadas y Google Scholar, y una vez revisada la literatura se obtiene un Marco Teórico.

El diseño del instrumento utilizado en la presente investigación fue construido en función de la literatura, con el propósito de plasmar los ítems o batería de reactivos que se adecuen a las variables que se obtienen en la hipótesis de estudio, es decir cada ítems fue seleccionado para obtener información que pueda validar los factores del modelo propuesto, lo anterior es justificado, ya que según Vara (2012) manifiesta que "mientras más instrumentos tengas, más fiable será; pues podrás seleccionar los mejores o hacer un híbrido entre ellos" (p. 270). Dicho instrumento tendrá variables de control y las variables de estudio serán dicotómicas, de selección y contendrán una escala de Likert del 1 al 5 para cada ítem en donde 1 es nada, 2 poco, 3 medio, 4 mucho, 5 demasiado.

Algunas variables de control son significativas y positivamente conectadas con la agilidad de la empresa como el dinamismo tecnológico percibido en la empresa (Mitręga, 2019). La variable dependiente, o llamada variable efecto (Vara, 2012), es el plan de marketing digital como lo refieren Lozano y Torres (2017), debe ser una guía clara con acciones definidas para los cumplir los deseos del cliente, con adopción en TIC de vanguardia mencionado por Amoah \& Bashiru (2021), como herramienta innovadora para la publicidad y la comunicación de marketing.

Como variables independientes o llamada variable causal (Vara, 2012), como lo refieren Sánchez y Arroyo (2016), la adopción de la tecnología, como variable se utiliza para estudiar el comportamiento ante la 
intención de una conducta mediante la actitud del individuo, afectada por la utilidad percibida y la facilidad de uso. El impacto como variable en la gestión, se emplea para conocer el efecto del marketing (Fernández, Esteves, Durand y Núñez, 2019) y la variable implementación es para llevar a cabo la utilización del uso de herramientas y funcionalidades (Martínez, 2018).

El instrumento consta de 5 secciones: los datos sociodemográficos, la influencia del marketing en la adopción de herramientas de vanguardia, el impacto del marketing digital, la planeación del marketing digital estratégico y los resultados de la implementación del marketing digital en cuanto a la rentabilidad.

Se analiza el instrumento con la técnica de expertos y su índice (K) "conocimiento", posteriormente se hará una prueba de pilotaje con una muestra representativa la cual se utilizará para realizar un pilotaje con $n=20 \mathrm{y}$ con ello procesar su base de datos y ver su fiabilidad y validez por medio del alfa de Cronbach.

Después se realiza el diagnóstico sobre el plan de marketing digital estratégico, por medio de un instrumento de investigación aplicado en línea a la muestra representativa del pilotaje, esto por medio de la herramienta Google Forms, en empresas del sector de comercio minorista (Glocker \& Piribauer, 2020) en Torreón, Coahuila, México.

Una vez aplicado el instrumento y estructurado la base de datos que contiene la información de las respuestas de cada uno de los ítems formulados se procederá a analizarlos por medio de la herramienta que permite procesar datos cuyo nombre es reconocido en las ciencias administrativas como software SPSS versión 19. Se procede a analizar los datos para la obtención de resultados y conclusiones.

\subsection{Métodos}

Con respecto al método utilizado en la compilación de los ítems del instrumento de investigación, se conformó tomando a consideración estudios previos que permiten solventar las variables de control y de estudio con sus respectivas mediciones que permitirán analizar posteriormente los factores que inciden sobre grado de adopción, el impacto y la adaptación de la implementación de un plan de marketing digital estratégico con adopción en TIC de vanguardia para posicionar a las Pyme del Sector Comercio, para ello Páez (2010) menciona que cuando sea posible medir hay que hacerlo de manera precisa mediante indicadores para una interpretación hacia la fiabilidad, para tal efecto se ha plasmado en la estructura del instrumento de investigación elementos que permiten dar una configuración de los ítems a partir de la literatura. Con el fin de obtener un instrumento confiable se revisó la configuración de los instrumentos de los siguientes autores como (Tajvidi \& Karami, 2021), (Sharma et al., 2020), (Lizama et al., 2020), (Krueger, 2019), (Brown et al., 2019), (Bautista, 2018), (Cant, 2016), (Valdez et al., 2014) de los cuales se extrajeron las dimensiones o bloques para construir un instrumento, es decir la información general del encuestado (datos sociodemográficos), influencia del marketing en adopción de herramientas de vanguardia, impacto del marketing digital, planeación de marketing digital estratégico y resultados de la implementación del marketing digital en cuanto a la rentabilidad, con preguntas que exigen respuestas dicotómicas utilizando para la elección una escala de Likert con cinco clasificaciones, es decir, del 0 al 4 , dando lugar a la valoración de parte del experto de los niveles de alcance del ítem en su respectiva dimensión $0=$ impacto menor y 4 $=$ impacto mayor.

3.2 Validación del instrumento sobre la adopción, el impacto y la adaptación de la implementación de un plan de marketing digital estratégico con adopción en TIC de vanguardia para posicionar a las Pyme del Sector Comercio por el método de Expertos 
El instrumento se sometió a depuración por el método de expertos en una variante utilizada por método Delphi (Sampieri, Valencia, y Soto, 2014), el cual se desarrolla en los siguientes pasos , (Michalus, Sarache, Hernández, Castro, y Pérez, 2015) y (Aguirre, Canibe y Jaramillo, 2019).

a) Identificación de las características a evaluar. Se determina el contexto de aplicación, así como los efectos y consecuencias de lo que se solicita evaluar, como parte de los antecedentes que se entregan al experto evaluador.

b) Selección de expertos en el área de la investigación. Este paso implica un proceso cuidadoso que permita la participación de expertos en el área de estudio, se integra un procedimiento de autoevaluación del propio experto propuesto por (Ramírez y Toledo 2005) y (Mesa 2007), citados por (Michalus et al., 2015).

Con base a los planteamientos de Mesa (2007) establece criterios para seleccionar a los expertos adecuadamente:

- Demostrar disposición a participar en la encuesta y contar con el tiempo para hacerlo.

- Disponer de una capacidad de análisis para comprender la problemática planteada y emitir un juicio confiable, así como capacidad prospectiva para analizar las situaciones que se podrían producir a partir de la aplicación de la solución propuesta.

- Poseer alta competencia (calculada a través del coeficiente de competencia K). La competencia de los expertos se determina calculando el coeficiente de competencia (K), según la expresión (1).

$K=1 / 2\left(K_{c}+K_{a}\right)$
Donde:

$\mathrm{K}_{\mathrm{c}}=$ coeficiente de conocimiento $\mathrm{o}$ información.

$\mathrm{K}_{\mathrm{a}}=$ coeficiente de argumentación $\mathrm{o}$ fundamentación.

El coeficiente de conocimiento o información $\mathrm{K}_{\mathrm{c}}$ se calcula sobre la base de la valoración del propio experto acerca del conocimiento o información que considera tener, acerca de los temas sobre los que se le consulta (problemática general y particular, conocimiento teórico y/o práctico, etc.), mediante una serie de preguntas que debe valorar en una escala de 0 a 10 puntos, donde el valor inferior (0) indica absoluto desconocimiento, y el superior (10), el pleno conocimiento de la referida problemática (Córdova, 2004) citados por (Michalus et al., 2015). Luego, el coeficiente $K_{c}$ de cada experto se calcula mediante la expresión (2).

$K_{c}=\frac{\sum_{i=1}^{i=n} K_{p i}}{10 . n}$

Donde:

$\mathrm{K}_{\mathrm{pi}}=$ autovaloración del experto sobre la pregunta pi del cuestionario.

$n=$ número total de preguntas del cuestionario de autovaloración.

El coeficiente de argumentación o fundamentación $\left(\mathrm{K}_{\mathrm{a}}\right)$ de los criterios del experto, es determinado como el resultado de la puntuación que el propio experto asigna a las principales fuentes de conocimiento en sus respuestas.

Se propone una tabla con las fuentes indicadas en las filas (ver Tabla 2), en donde cada experto debe indicar el grado de influencia de dicha fuente en sus conocimientos declarados sobre el tema, de acuerdo con los niveles Alto (A), Medio (M) y Bajo (B). Se calcula entonces $K_{a}$ como la suma de los puntos a partir de comparar las casillas marcadas por el experto, donde se establece a priori la puntuación asignada a cada fuente. 
Finalmente, se calcula el coeficiente de competencia K según la expresión (1) y se evalúa la competencia del experto mediante la aplicación de la escala siguiente:

-Si: $0,80<\mathrm{K} \leq 1,00$; se considera que el candidato tiene una competencia Alta.

-Si: $0,50<\mathrm{K} \leq 0,80$; se considera que el candidato tiene una competencia Media.

- Si: $\mathrm{K} \leq 0,50$; se considera que el candidato tiene competencia Baja; si fuera este el caso, entonces se descarta como experto. Finalmente, en este paso, se seleccionan todos los candidatos a expertos cuyo coeficiente de competencia K sea Alto.

c) Diseño del instrumento de recolección de datos.

d) Consulta de expertos, se envía el cuestionario a evaluar y los instrumentos de recolección de datos, así como los propósitos y objetivos de dicha evaluación.

e) Procesamiento de la información, con el análisis de los resultados y sugerencias.

f) Elaboración de conclusiones.

Tabla 1 Test de Autoevaluación para el experto para el cálculo del Coeficiente de información (Kc).

\begin{tabular}{l}
\hline Relación de atributos o características \\
para cálculo de Kc \\
\hline Conocimiento \\
Competitividad \\
Disposición \\
Creatividad \\
Profesionalidad \\
Capacidad de análisis 1 y 10 \\
Experiencia \\
Intuición \\
Actualización \\
Capacidad de trabajo en equipo \\
\hline Fuente: (Torrado-Fonseca, 2016) y (Aguirre et al., 2019)
\end{tabular}

Tabla 2 Test de Autoevaluación del experto para determinar el coeficiente de Argumentación (Ka).

\begin{tabular}{|c|c|c|c|c|}
\hline No. & Fuentes de Argumentación & $\begin{array}{l}\text { Alto } \\
\text { (A) }\end{array}$ & $\begin{array}{l}\text { Medio } \\
\text { (B) }\end{array}$ & $\begin{array}{l}\text { Bajo } \\
\text { (C) }\end{array}$ \\
\hline \multirow{6}{*}{$\mathrm{C}$} & Análisis teóricos realizados & & & \\
\hline & Experiencia que posee en estos temas & & & \\
\hline & Conocimiento de trabajo de autores nacionales & & & \\
\hline & Conocimiento de trabajo de autores extranjeros & & & \\
\hline & Consultas bibliográficas de estos temas & & & \\
\hline & Cursos de actualización & & & \\
\hline
\end{tabular}

3.3 Validación del instrumento de Autoevaluación sobre una propuesta de plan de marketing digital estratégico con adopción en TIC de vanguardia para posicionar a las Pymes Comercio por el método de Alpha de Cronbach.

La fiabilidad se relaciona con la precisión y congruencia, es el grado en que la aplicación repetida de un instrumento al mismo sujeto, objeto o situación produce iguales resultados 
"La confiabilidad de un instrumento de medición se refiere al grado en que su aplicación repetida al mismo individuo $u$ objeto produce resultados iguales" (Hernández et al., 2014, p.200). Además, es la capacidad del instrumento de producir resultados congruentes (iguales), cuando se aplica por segunda o tercera vez, en condiciones tan parecidas como sea posible (Vara, 2012).

"Para saber si un instrumento es confiable a partir de las covarianzas y la consistencia interna, se requiere establecer si las variaciones pueden comportarse en un recorrido de múltiples categorías que no exista una opción para clasificar respuestas correctas e incorrectas o si cada ítem puede recibir respuestas dicotómicas o de dos categorías, lo que incluye aquellos ítems de múltiple opciones pero con respuestas correctas y las demás incorrectas" (Martínez \& March, 2015, p. 119).

Para valorar un instrumento de acuerdo con Jorna, Castañeda y Véliz (2015) se guían bajo los criterios de Moriyama:

Razonable y comprensible. Que cada pregunta tenga relación con el tema que se pretende medir.

Sensible a variaciones en el fenómeno que se mide. Si el instrumento puede derivare un índice que muestren diferencias en la variable que medirá.

Con suposiciones básicas justificables in intuitivamente razonables. Que tenga una razón de ser cada pregunta.

\section{RESULTADOS}

Para la obtención de los resultados se revisaron la configuración de los instrumentos de los siguientes autores como (Tajvidi \& Karami, 2021), (Sharma et al., 2020), (Lizama et al., 2020), (Krueger, 2019), (Brown et al., 2019), (Bautista, 2018), (Cant, 2016), (Valdez et al., 2014) donde se obtuvieron 5 bloques los datos sociodemográficos, la influencia del
Con componentes claramente definidos: que cada pregunta sea sencilla de comprender Derivable de datos factibles de obtener: a partir de los ítems tratar de obtener las respuestas deseadas.

La validez, por su parte, es el grado en que un instrumento realmente mide la variable que pretende medir. La validez se refiere al grado de evidencia acumulada que mide el instrumento, justifica la particular interpretación que se va a hacer del instrumento (Vara, 2012). Como también lo refieren Hernández et al. (2014) que "La validez, en términos generales, se refiere al grado en que un instrumento mide realmente la variable que pretende medir" (p. 200).

El coeficiente Alfa de Cronbach es un modelo de consistencia interna, basado en el promedio de las correlaciones entre los ítems. Entre las ventajas de esta medida se encuentra la posibilidad de evaluar cuánto mejoraría (o empeoraría) la fiabilidad de la prueba si se excluyera un determinado ítem.

El método utilizado para determinar el coeficiente del Alfa de Cronbach se desarrolla por los siguientes pasos:

- Se parte de la elaboración del instrumento.

- Se aplica una prueba piloto

- Se capturan los datos en un software estadístico

- Se calcula el Alfa de Cronbach

- Se analizan los resultados

marketing en la adopción de herramientas de vanguardia, el impacto del marketing digital mediante la cultura de la adopción de herramientas de vanguardia, la implicación de la planeación del marketing digital estratégico y la implementación del marketing digital en cuanto a la rentabilidad con un total de 82 ítems con diseño de respuestas dicotómicas o escala de Likert. Se muestran las dimensiones definidas para el cuestionario de evaluación en 
la Tabla 3.

Tabla 3 Dimensiones definidas para el cuestionario de autoevaluación

\begin{tabular}{|c|c|}
\hline DIMENSIÓN & DESCRIPCIÓN \\
\hline Datos sociodemográficos & $\begin{array}{l}\text { Recaba datos de identificación como: género, edad, escolaridad, } \\
\text { ocupación, número de empleados, antigüedad de la empresa, } \\
\text { actividad económica principal. }\end{array}$ \\
\hline $\begin{array}{llll}\text { Influencia del marketing en la } \\
\text { adopción de herramientas de } \\
\text { vanguardia }\end{array}$ & $\begin{array}{l}\text { Mide aspectos relacionados con la promoción, la consideración de } \\
\text { plataformas en los negocios, las barreras que supone el utilizar el } \\
\text { marketing digital. }\end{array}$ \\
\hline Impacto del marketing digital & $\begin{array}{l}\text { Mide los criterios para conocer la utilización de medios digitales, } \\
\text { cuales utiliza y que le traen en consecuencia. }\end{array}$ \\
\hline $\begin{array}{l}\text { La planeación del marketing digital } \\
\text { estratégico. }\end{array}$ & $\begin{array}{l}\text { Se observa si planean, el tiempo que se invierte, las desventajas, quien } \\
\text { maneja las redes. }\end{array}$ \\
\hline $\begin{array}{l}\text { Resultados de la implementación del } \\
\text { marketing digital en cuanto a la } \\
\text { rentabilidad }\end{array}$ & $\begin{array}{l}\text { Mediante la implementación del marketing digital se mide el } \\
\text { crecimiento, rentabilidad, retorno de la inversión, porcentaje de } \\
\text { crecimiento. }\end{array}$ \\
\hline
\end{tabular}

Para analizar por el método de expertos, se solicitó a investigadores conocedores del expertis mediante la evaluación de atributos y tema, quienes se autoevaluaron en su grado de características para determinar el valor del coeficiente de conocimiento $\mathrm{K}$ ver tabla 4.

Tabla 4 Atributos o características para el cálculo de Kc

\begin{tabular}{lc}
\hline Relación de atributos o características & Selección entre 1 y 10 \\
para cálculo de Kc & 9 \\
\hline Conocimiento & 9 \\
Competitividad & 9 \\
Disposición & 10 \\
Creatividad & 9 \\
Profesionalidad & 9 \\
Capacidad de análisis & 9 \\
Experiencia & 10 \\
Intuición & 9 \\
Actualización & 10 \\
Capacidad de trabajo en equipo &
\end{tabular}

Se aprecian los valores tomados de uno de los expertos sobre el tema de investigación, para

la determinación de valores de Argumentación (ka) de expertos. Ver Tabla 5.

Tabla 5 Fuente de argumentación para Ka

\begin{tabular}{|c|c|c|c|c|}
\hline $\begin{array}{l}\text { No. } \\
\text { (n) }\end{array}$ & Fuentes de Argumentación & $\begin{array}{l}\text { Alto } \\
\mathrm{A}=1 \\
\mathrm{Ka} 1\end{array}$ & $\begin{array}{c}\text { Medio } \\
\mathrm{B}=0.5 \\
\mathrm{ka} 2\end{array}$ & $\begin{array}{c}\text { Bajo } \\
\text { (C) }=0 \\
\text { Ka3 }\end{array}$ \\
\hline 1 & cos realizados & & B & \\
\hline
\end{tabular}


2 Experiencia que posee en estos temas

B

3 Conocimiento de trabajo de autores nacionales A

4 Conocimiento de trabajo de autores extranjeros A

5 Consultas bibliográficas de estos temas A

6 Cursos de actualización A

Total de puntaje: 5.00

Elaboración propia.

$\frac{1}{2}(1.76)=\mathbf{0 . 8 8}$

$K_{C}=\frac{\sum_{i=1}^{i=n} K_{i}}{10 . n}=\frac{\sum 9,9,10,9,9,9,10,9,10}{10.10}=\frac{0.9333}{100}=\quad$ El resultado para el experto analizado es de

0.93 0.88

$K_{c}=\frac{\sum(3+1)}{6}=-\frac{5}{6}=\mathbf{0 . 8 3}$

$\mathrm{K}=\frac{1}{2}(K c+K a)=\frac{1}{2}(0.93+0.83)=$

Tomando en cuenta los valores de $\mathrm{K}$ para determinar el nivel de competencia se tiene la Tabla 6.

Tabla 6 Expertos y su nivel de competencia

\begin{tabular}{|c|c|c|c|c|c|}
\hline No. & Experto & $\mathrm{Kc}$ & $\mathrm{Ka}$ & $\mathrm{K}$ & $\begin{array}{l}\text { Nivel de } \\
\text { competencia }\end{array}$ \\
\hline 1 & Doctor & 0.77 & 0.75 & 0.76 & medio \\
\hline 2 & Maestría & 0.50 & 1.00 & 0.75 & medio \\
\hline 3 & Maestría & 0.88 & 0.33 & 0.61 & medio \\
\hline 4 & Maestría & 0.96 & 0.50 & 0.73 & medio \\
\hline 5 & Doctorante & 0.74 & 0.50 & 0.62 & medio \\
\hline 6 & Maestría & 0.82 & 0.83 & 0.83 & alta \\
\hline 7 & Doctorante & 0.93 & 0.83 & 0.88 & alta \\
\hline 8 & Maestría & 0.62 & 0.83 & 0.73 & medio \\
\hline 9 & Doctorante & 0.90 & 0.50 & 0.70 & medio \\
\hline 10 & Maestría & 0.70 & 0.25 & 0.48 & bajo \\
\hline 11 & Maestría & 0.57 & 0.17 & 0.37 & bajo \\
\hline 12 & Doctorante & 0.90 & 1.00 & 0.95 & alta \\
\hline 13 & Doctorante & 1.00 & 1.00 & 1.00 & alta \\
\hline 14 & Doctorante & 0.87 & 0.33 & 0.60 & medio \\
\hline 15 & Maestría & 0.93 & 0.17 & 0.55 & medio \\
\hline 16 & Doctorante & 1.00 & 0.58 & 0.79 & medio \\
\hline 17 & Doctorante & 0.80 & 0.50 & 0.65 & medio \\
\hline 18 & Maestría & 0.49 & 0.50 & 0.50 & bajo \\
\hline 19 & Doctorante & 0.64 & 0.67 & 0.65 & medio \\
\hline 20 & Doctorante & 0.86 & 0.17 & 0.51 & medio \\
\hline 21 & Maestría & 0.50 & 0.17 & 0.33 & bajo \\
\hline
\end{tabular}

Elaboración Propia.

En base a Dueñas, Medina, Ramírez, Camacho

con $(0.8 \leq \mathrm{K} \leq 1)$, de alta competencia y $(0.5$ y Sobenis et al. (2019) selecciona expertos $\leq \mathrm{K}<0.8)$ de media competencia, por su parte 
Martínez et al. (2018) seleccionan expertos con índice $\mathrm{K}$ alto y medio. Debido a lo anterior, se seleccionaron 4 expertos con un nivel de competencia alto y 6 de nivel medio se ve en la Tabla 6.

Tabla 7. Valores obtenidos en Alfa de Cronbach del instrumento completo y por dimensiones o bloques.

\begin{tabular}{lccl}
\hline Dimensión/Bloques/ Elementos & Alfa de Cronbach & Nivel de Fiabilidad \\
\hline Todas $-82-4=78$ & 0.974 & Muy bueno \\
Influencia del marketing en adopción de herramientas de & 0.873 & Bueno \\
$\begin{array}{l}\text { vanguardia. } \\
\text { Impacto del marketing digital. }\end{array}$ & 0.978 & Muy bueno \\
Planeación de marketing digital estratégico. & 0.737 & Aceptable \\
$\begin{array}{l}\text { Resultados de la implementación del marketing digital en cuanto a } \\
\text { la rentabilidad. }\end{array}$ & 0.880 & Bueno \\
\hline
\end{tabular}

Elaboración Propia.

\section{CONCLUSIONES}

El objetivo del presente estudio ha sido la validación del instrumento de investigación para determinar el grado de adopción, el impacto y la adaptación de la implementación de un plan de marketing digital estratégico con adopción en TIC de vanguardia para posicionar a las Pyme del Sector Comercio.

Para tal efecto, se recurrió a determinar la confiabilidad del instrumento, después de haber lo sometido al método de expertos, se procedió a utilizar el método de Alfa de Cronbach, dentro de las secciones del instrumento, en donde al bloque IV tuvo un resultado débil de 0.607 , respecto a su nivel de fiabilidad, por lo tanto se le aplico el total estadístico elemento, por lo tanto se omite la pregunta ¿la empresa cuenta con un plan anual sobre el uso de redes, alineados con los objetivos generales de la empresa? con lo que el Alfa de Cronbach sube a 0.646, al omitir la siguiente pregunta ¿Qué persona maneja el manejo de redes? el Alfa de Cronbach sube a 0.682, después al quitar la pregunta ¿Cuánto tiempo invierte en el marketing digital, excluyendo el uso del mail? aumenta a 0.715, y por último al eliminar la pregunta ¿En qué grado considera que hay desventajas en el uso de redes sociales para su negocio? [distracción de empleados], da un total de 0.737 por lo cual de los 12 ítems se le quitaron 4, quedando 8 ítems que resulta dentro de un nivel aceptable. En lo referente al Bloque II Influencia del marketing en adopción de herramientas de vanguardia con un nivel de confiabilidad bueno de 0.873 , en lo que respecta al Bloque III Impacto del marketing digital con un nivel de confiabilidad muy bueno de 0.978 , y el Bloque V Resultados de la implementación del marketing digital en cuanto a la rentabilidad con un nivel de fiabilidad bueno de 0.880 , quedando el instrumento con un total de 78 ítems con un total de 0.974 . Ver Tabla 7.

El presente estudio genera contribuciones debido a lo anterior, ya que el instrumento puede ser utilizado al aplicarlo con una muestra mayor y obtener resultados sobre la planeación del marketing digital estratégico con adopción en TIC de vanguardia para posicionar a las Pymes Comercio, ya que basado en el promedio de las 
correlaciones entre los ítems muestra consistencia interna, es decir, muestra reproductibilidad, seguridad y precisión, el cual se le conoce como confiabilidad (Vara, 2012), al ser aplicado este instrumento hay evidencia estadística para lograr la confianza de que se contará con información fiable y válida en los resultados.

Adicionalmente, el estudio también tiene implicaciones futuras, ya que es importante mencionar que se realizará en un futuro mediato la aplicación de este instrumento obtenido con la literatura y validado con la herramienta estadista, por tal en un próximo estudio se buscara realizar un análisis factorial exploratorio (AFE) para dar validez a los constructos y con ello diseñar y proponer un modelo con los factores e indicadores que impactan en un plan de marketing digital estratégico con adopción en TIC de vanguardia para posicionar a las Pymes Comercio en Torreón, Coahuila. 


\section{REFERENCIAS}

Aguirre, E., Canibe, F., y Jaramillo, M. (2019). Validación de cuestionario de Autoevaluación de Competencias para el docente virtual universitario. Vinculatégica, (5)(Junio), 215-228. http//www.web.facpya.unal.mx/vinculategica/.

Alameddine, A. (2013). Perceptions of Executives from seven selected Companies of the Use of Social Media in Marketing Practices. Pepperdine University Graduate School of Education and Psychology, Graduate School of Education and Psychology, 122.

Álvarez, J. (2021). Digital Report 2021: El informe sobre las tendencias digitales, redes sociales y mobile. Wearesocial. https://wearesocial.com/es/blog/2021/01/digital-report-2021-el-informesobre-las-tendencias-digitales-redes-sociales-y-mobile

Amoah, J., \& Bashiru, A. (2021). Inhibitors of social media as an innovative tool for adverstising and marketing communication: evidence from SMES in a developing country. Innovative Marketing, 15(4), 164-179. https://doi.org/http://dx.doi.org/10.21511/im.16(4).2020.15

Angamarca, M., Mena, D., Silva, F., y Ballesteros, L. (2019). Estrategias de Marketing Digital en Empresas E-Commerce: Un acercamiento a la perspectiva del consumidor. Digital Publisher CEIT, 4(51). https://doi.org/https://doi.org/10.33386/593dp.2019.5-1

Arellano, J., Romero, S., y Guzmán, G. (2018). Marketing digital y las finanzas de las Pymes. RITI Journal, 6.

Armijos, N. (2018). Marketing digital: una herramienta para potenciar y promocionar las Mypimes en el ámbito internacional. INNOVA Research Journal. https://doi.org/doi.org/10.33890/innova.v4.n1.2019.764

Bautista, G. E. (2018). La valoración del Community Manager en el Marketing Digital de PyMes de la Ciudad de Xalapa, Ver. - Conacyt.

Brown, D., Foroudi, P., \& Hafeez, K. (2019). Marketing management capability: the construct and its dimensions An examination of manager's and entrepreneurs ' perceptions in a retail setting. Quealitative Market Reserarch: An International Journal, 22(5), 609-637. https://doi.org/10.1108/QMR-10-2017-0131

Calle, K., Erazo, J., y Narváez, C. (2020). Marketing digital y estrategias online en el sector de fabricación de muebles de madera. Revista Arbitrada Interdisciplinaria Koinonía, 5(10), 339. https://doi.org/10.35381/r.k.v5i10.698

Cant, M. (2016). Using social media to market a promotional event to SMEs: opportunity or wasted effort? Problems and Perspectives in Management, 14(4), 76-82. https://doi.org/10.21511/ppm.14(4).2016.09

Cates, D. (2018). Small Business Marketing Analytics Capitol Technology University. ProQuest 28262220.

Correa, L. Á. (2019). Relacion entre la tecnologia y la comercialización en la PyME. Mercados y Negocios Dialnet, 1(41).

Dirgiatmo, Y. (2015). Analysis of the Potential Use of Social Networking for the Success of Strategic Business Planning in Small and Medium-Sized Enterprises. Mediterranean Journal of Social Sciences, 6(2), 233-245. https://doi.org/10.5901/mjss.2015.v6n2s2p233

Dueñas, J., Medina, A., Ramírez, L., Camacho, W., y Sobenis, J. (2019). La prospectiva estratégica como herramienta de planeación a largo plazo. Magazine de Las Ciencias, 4(3), 1-18. https://doi.org/10.5281/zenodo.3339463

Escobar, J., y Cuervo, Á. (2008). Validez de contenido y juicio de expertos: Una paroximación a su utilización. Avances En Medición, 6, 27-36. https://www.researchgate.net/publication/302438451_Validez_de_contenido_y_juicio_de_exp ertos_Una_aproximacion_a_su_utilizacion

Fernández, V., Esteves, A., Durand, J., y Núñez, H. (2019). Habilidades Blandas Y Su Impacto En La Gestión De La Mezcla De Marketing. Un Estudio Realizado En El Emporio Comercial Gamarra, Perú. In Revista Científica ECOCIENCIA (Vol. 6, Issue 2).

Galeano, S. (2021). Cuáles son las redes sociales con más usuarios del mundo 2021. M4rketing 
Ecommerce. https://marketing4ecommerce.net/cuales-redes-sociales-con-mas-usuariosmundo-ranking/

García, M. (2021). Redes Sociales en pandemia muestran crecimiento acelerado. Mauna Media Inbound y Marketing Digital Estratégico. https://www.maunamedia.com/redes-sociales-enpandemia-muestran-crecimiento-acelerado/\#.YCKi-uhKi00

Glocker, C., \& Piribauer, P. (2020). Digitalization, retail trade and monetary policy. Journal of International Money and Finance, 102340. https://doi.org/10.1016/j.jimonfin.2020.102340

González, C., y Moreno, K. (2020). Social media como estrategia de posicionamiento de marca de PYMES textiles de la provincia de Tungurahua. Digital Publisher CEIT, 5(3), 49-60. https://doi.org/doi.org/10.33386/593dp.2020.3.209

Grandhi, B., Patwa, N., \& Saleem, K. (2020). Data-driven marketing for growth and profitability. EuroMed Journal of Business, ahead-of-p(ahead-of-print). https://doi.org/10.1108/EMJB-092018-0054

Gupta, S., \& Chokshi, S. (2020). Digital Marketing Effectiveness Using Incrementality. In Communications in Computer and Information Science: Vol. 1244 CCIS. https://doi.org/10.1007/978-981-15-6634-9_10

Hänninen, M., Kwan, S. K., \& Mitronen, L. (2021). From the store to omnichannel retail : looking back over three decades of research. The International Review of Retail, Distribution and Consumer Research, 31(1), 1-35. https://doi.org/10.1080/09593969.2020.1833961

Hernández, R, Fernández, C., y Baptista, P. (2014). Metodología de la Investigación. Sexta Edición. In Mc Graw Hill.

Hernández, Roberto, Méndez, S., y Contreras, R. (2014). Construcción de un instrumento para medir el clima organizacional en función del modelo de los valores en competencia. Contaduría y Administración, 59(1), 229-257. https://doi.org/10.1016/S0186-1042(14)71250-1

Hoyos, S., y Sastoque, J. (2020). Marketing Digital como oportunidad de digitalización de las PYMES en Colombia en tiempo del Covid - 19. Revista Científica Anfibios, 3(1), 39-46. https://doi.org/10.37979/afb.2020v3n1.60

Jaramillo, S., Tacuri, L., y Trelles, D. (2018). Planeación estratégica de marketing y su importancia en las empresas. INNOVA Research Journal, 3(10.1), 28-41. https://doi.org/10.33890/innova.v3.n10.1.2018.737

Jorna, A., Castañeda, I., y Véliz, P. (2015). Construcción y validación de instrumentos para directivos de salud desde la perspectiva de género Construction and validation of instruments for executives of health from the perspective of gender Introducción. Horizonnte Sanitario, 14(3), 101-110. https://www.redalyc.org/pdf/4578/457844966005.pdf

Juárez, E. (2017). Importancia del marketing en las PYMES. II Congreso Virtual Internacional Desarrollo Económico, Social y Empresarial en Iberoamérica (Servicios).

Junusi, R. (2020). Digital Marketing During the Pandemic Period; A Study of Islamic Perspective. Journal of Digital Marketing and Halal Industry. https://doi.org/10.21580/jdmhi.2020.2.1.5717

Kato, E. (2019). Productividad e innovación en pequeñas y medianas empresas. Estudios Gerenciales Journal of Management and Economics of Iberoamerica, 35(150), 38-46. https://doi.org/https://doi. org/10.18046/j.estger.2019.150.2909

Kaur, J. (2020). Influence of technological advances and change in marketing strategies using analytics in retail industry. International Journal of System Assurance Engineering and Management. https://doi.org/10.1007/s13198-020-01023-5

Khajehasani, S., Abolizadeh, A., \& Dehyadegari, L. (2020). The Role of Management and Strategy in the Development of E-Marketing. Recent Advances in Computer Science and Communications, 13(4), 641-649. https://doi.org/10.2174/2213275912666190411114639

Krueger, G. (2019). Microbusiness marketing with no time, no money, and no expertise research and recommendations for improving marketing results from microbusiness. ProQuest Number:13890241, January. https://pqdtopen.proquest.com/doc/2211608337.html?FMT=AI

Lizama, Y., Matos, L., y Beltrán, R. (2020). Online marketing: rentabilidad al alcance de las 
Mipymes. Revista Visión $\quad$ Contable, $\quad 21, \quad 57-76$. https://doi.org/https://doi.org/10.24142/rvc.n21a3 Resumen

Londoño, S., Mora, Y., y Valencia, M. (2018). Modelos estadísticos sobre la eficacia del marketing digital. Revista EAN, 84, 167-186. https://doi.org/https://doi.org/10.21158/01208160.n84.2018.192

Lozano, E., y Torres, G. (2017). Modelo práctico de plan estratégico de mercadotecnica para micro y pequeñas empresas de transformación en Lagos de Moreno, Jalisco. Ra Ximhai, 13(3), 405416. https://www.redalyc.org/articulo.oa?id=461/46154070024

Madrigal, S., Alfaro, G., y Madrigal, F. (2018). Redes sociales virtuales en México, área de oportunidad para la organización contemporánea. Razón y Palabra, 22(100), 856-872. https://www.revistarazonypalabra.org/index.php/ryp/article/view/1139

Maloletko, A., \& Kaurova, O. (2021). Frontier Information Technology and Systems Research in Cooperative Economics (A. V. Bogoviz, A. E. Suglobov, A. N. Maloletko, O. V. Kaurova, \& S. V. Lobova (eds.); Vol. 316). Springer International Publishing. https://doi.org/10.1007/9783-030-57831-2

Maridueña, A., y Paredes, J. (2015). Plan de Marketing Digital 2014 para la empresa Corporación de Servicios TBL $S$. A . de la ciudad de Guayaquil [Ecuador]. https://dspace.ups.edu.ec/bitstream/123456789/9939/1/UPS-GT000974.pdf

Martínez, A. (2018). Marketing 2.0 aplicado al sector turístico: la Función comercial de los sitios Web de las organizaciones de marketing destinos. Vivat Academia Revista de Comunicación, 143, 01-23. https://doi.org/http:/ //doi.org/ 0.15178/va.2018.143.0 1 01-23

Martínez, E., Travieso, N., Sagaró, N., Urbina, O., y Martínez, I. (2018). Validación de competencias específicas de los profesionales de enfermería en la atención al neonato en estado grave. Medisan, 22(3), 295-303.

Martínez, M., y March, T. (2015). Caracterización de la validez y confiabilidad en el costructo metodológico de la Investigación Social. REDHECS Revista Electrónica de Humanidades, Educación y Comunicación Social, 20(10), 107-127.

Mejía, J. (2019). Cómo se relaciona la innovación en la mercadotecnia digital y el e-liderazgo en las PYMES. Revista de El Colegio de San Luis, 9(20), 77-111. https://doi.org/DOI https://doi.org/10.21696/rcs19202019948

Michalus, J. C., Sarache, W. A., Hernández, G., Castro, W. A. S., y Pérez, G. H. (2015). Metodo de expertos para la evaluación ex-ante de una solución organizativa. Visión de Futuro, 19(1), 117.

Mitręga, M. (2019). Dynamic marketing capability - refining the concept and applying it to company innovations. Journal of Business \& Industrial Marketing, 35(2), 193-203. https://doi.org/10.1108/JBIM-01-2019-0007

Morán, C., y Cañarte, T. (2017). Redes sociales factor predominante en la fidelización del cliente. Dominio de Las Ciencias, 3(4), 519-532. https://doi.org/http://dx.doi.org/10.23857/dom.cien.pocaip.2017.3.núm. 4.oct.519-532

More, R., Castillo, F., y Rodríguez, N. (2018). Uso Del Comercio Electrónico Aplicando El Modelo De Aceptación Tecnológica ( Tam ) En Profesionales Universitarios Piura-Perú. ResearchGate. https://www.researchgate.net/publication/331385867_USO_DEL_COMERCIO_ELECTRON ICO_APLICANDO_EL_MODELO_DE_ACEPTACION_TECNOLOGICA_TAM_EN_PRO FESIONALES_UNIVERSITARIOS_PIURA-PERU_2018

Narváez, G., \& Montalvo, E. (2014). Best Practice in the Use of Social Networks Marketing Strategy as in SMEs. Procedia - Social and Behavioral Sciences, 148(443), 533-542. https://doi.org/10.1016/j.sbspro.2014.07.076

Noreña, Y., y Álvarez, L. (2020). Evaluación de las estrategias de marketing en el restaurante Yukis en la ciudad de Puyo. Ingeniería En Administración de Empresas y Negocios. http://dspace.uniandes.edu.ec/handle/123456789/11347

Núñez, C., y Corrales, M. (2020). El marketing digital como un elemento de apoyo estratégico a las 
organizaciones. Cuadernos Lationoamericanos de Administración, XVI(30), 1-14. https://doi.org/10.18270/cuaderlam.v16i30.2915

Oquendo, S. (2016). Fides et Ratio - Revista de Difusión cultural y científica de la Universidad La Salle en Bolivia Auditoria de marketing como instrumento de evaluación de las estrategias comerciales de las empresas. Revista de Difusión Cultural y Científica de La Universidad La Salle En Bolivia, 1-11. http://www.scielo.org.bo/scielo.php?pid=s2071081x2016000100012\&script=sci_arttext

Páez, O. (2010). Evaluación de las funciones docentes en entornos educativos virtuales (EIV), certezas, cuentionamiento y sincerameinto. Revista Iberoamericana de Evaluación Educativa, $3(1)$.

Pangemanan, S. A., \& Walukow, I. M. (2018). Marketing Strategy Analysis for Small and Medium Scale Business Enterprise (SMEs) for Home Industry Furniture in Leilem, the Regency of Minahasa. Journal of Physics: Conference Series, 953(1), 012033. https://doi.org/10.1088/1742-6596/953/1/012033

Phippen, A., Sheppard, L., \& Furnell, S. (2004). A practical evaluation of Web analytics. Internet Research, 14(4), 284-293. https://doi.org/10.1108/10662240410555306

Sánchez, C., y Tena, A. (2019). Social Media: Desarrollo Del Plan De Social Media De Una Empresa. 62.

Sánchez, E., García, J., \& Rodríguez, M. (2020). The Application of the Inbound Marketing Strategy on Costa del Sol Planning \& Tourism Board. Lessons for Post-COVID-19 Revival. Sustainability, 12(23), 9926. https://doi.org/10.3390/su12239926

Sánchez, J., y Arroyo, F. (2016). Diferencias de la adopción del comercio electrónico entre países. Suma de Negocios, 7, 141-150. https://doi.org/10.1016/j.sumneg.2016.02.008

Segarra, J., Martínez, A., y Gauchi, J. (2018). Planificación estratégica de la comunicación integrada. $\begin{array}{lllll}\text { Razón } y & \text { Palabra, 22(3), } & 152-178 .\end{array}$ http://www.revistarazonypalabra.org/index.php/ryp/article/view/1263/1282

Sharma, S., Singh, S., Kujur, F., \& Das, G. (2020). Social Media Activities and Its Influence on Customer-Brand Relationship: An Empirical Study of Apparel Retailers' Activity in India. Journal of Theoretical and Applied Electronic Commerce Research, 16(4), 602-617. https://doi.org/10.3390/jtaer16040036

Stanton, W. (1987). Fundamentos de mercadotecnica (McMcGraw H). McGraw Hill.

Taiminen, H., \& Karjaluoto, H. (2015). The usage of digital marketing channels in SMEs. Journal of Small Business and Enterprise Development, 22(4), 633-651. https://doi.org/10.1108/JSBED05-2013-0073

Tajvidi, R., \& Karami, A. (2021). The effect of social media on firm performance. Computers in Human Behavior, 115(August), 105174. https://doi.org/10.1016/j.chb.2017.09.026

Terán, O., Espinosa, E., Márquez, O., Hernández, P., Ruiz, E., Vergara, M., y Lara, M. (2018). Inbound Marketing como estrategia competitiva para el comercio detallista de las Mipymes en el Municipio de Juchitepec, Estado de México. Revista Global de Negocios, 6(7), 87-97. http://www.theibfr2.com/RePEc/ibf/rgnego/rgn-v6n7-2018/RGN-V6N7-2018.pdf\#page=89

Torradoa, M., y Reguant, M. (2016). El metodo Delphi. REIRE. Revista d'Innovació i Recerca En Educació, 9(1), 87-102. https://doi.org/10.1344/reire2016.9.1916

Torres, R., Rivera, J., Cabarcas, R., y Castro, Y. (2017). La efectividad del uso del marketing digital como estrategia para el posicionamiento de las pymes para el sector comercio en la ciudad de Barranquilla. Revista I+D En TIC, 8(2), 3-5.

Valdez, L., Rascón, J., Ramos, E., y Huerta, J. (2014). Redes Sociales, una Estrategia Corporativa para las PyMES de la región de Guaymas Sonora México. Revista FIR, FEADPYME International Review, 1(1), 62-74. https://doi.org/10.15558/fir.v1i1.15

Vara, A. (2012). 7 Pasos para una tesis exitosa. Desde la idea inicial hasta la sustentación. Un método efectivo para las ciencias empresariales. In Instituto de Investigación de la Facultad de Ciencias Administrativas y Recursos Humanos. Universidad de San Martín de Porres, Lima. 
Www.aristidesvara.net

Vidal, P. (2016). Metodología Para La Elaboración De Un Plan De Marketing Online. 3C Empresa $\begin{array}{lllll}\text { Investigación } \quad y & \text { Pensamiento } & \text { Crico, } & \text { 05(02), }\end{array}$ https://doi.org/10.17993/3cemp.2016.050226.57-72 